\title{
Etat des lieux de la pandémie de COVID-19 en Côte d'Ivoire
}

\section{Status of the COVID-19 pandemic in Côte d'Ivoire}

\author{
Kouame $\mathrm{KL}^{1^{*}}$, Yao $\mathrm{AB}^{2}$, N'Dri $\mathrm{KI}^{3}$. \\ 1. Ingénieur Génie Chimique et Procédés (IGCP)/ Chercheur en sciences des Procédés Chimiques Alimentaires et \\ Environnementaux à Ecole Doctorale Polytechnique (EDP)/ Institut National Polytechnique Houphouët-Boigny (INP-HB) de \\ Yamoussoukro (Côte d'Ivoire). \\ 2. Etudiante en Licence 3 Sage-Femme de l'Institut National de Formation des Agents de santé (INFAS) de Bouaké (Côte \\ d'Ivoire). \\ 3. Etudiant en Master 2 de Philosophie de l'Université Alassane Ouattara de Bouaké (Côte d'Ivoire).
}

Auteur correspondant : Kouamé Konan Lopez, Institut National Polytechnique Houphouët-Boigny (INP-HB) de Yamoussoukro (Côte d'Ivoire). Email : kouamekonanlopez@ gmail.com

\begin{abstract}
Résumé
Contexte: Le covid-19 (coronavirus disease - 19) est une infection causée par un coronavirus dit SARS-Cov_2 (Severe Acute Respiratory Syndrome Coronavirus 2), un virus très contagieux qui affecte les voies respiratoires. On en compte un grand nombre de patients dans le monde causant de nombreuses pertes en vies humaines. En Côte d'Ivoire comme partout ailleurs, les gouvernements et les spécialistes se sont engagés dans une dynamique de lutte contre le covid-19. Jusqu'à ce jour, le moyen de lutte par excellence reste la prévention.Objectif : Faire un état des lieux de la pandémie de covid-19 en Côte d'Ivoire en vue d'en améliorer la lutte.Méthodes : La première étape a consisté à l'analyse de la progression de la pandémie en Côte d'Ivoire sur la période de mars à mai 2020. La deuxième étape a été le diagnostic du mode de transmission de la pandémie. La troisième étape a consisté en la recherche des facteurs de risque à partir d'enquêtes de terrain et d'analyse des faits observés de façon générale en Côte d'Ivoire.Résultats : L'analyse de la progression de la pandémie montre que celle-ci se propage de façon aléatoire et à un rythme très élevé en Côte d'Ivoire. Le nombre de personnes infectées est inégalement réparti sur l'étendue du territoire national et dans le temps. Les résultats du diagnostic du mode de transmission de la pandémie en Côte d'Ivoire à partir de la carte de contrôle des moyennes ont montré que la transmission de la pandémie dans le pays est hors contrôle. La recherche des facteurs de risque à partir d'enquêtes de terrain et d'analyse des faits observés dans le pays révèle que plusieurs causes sont à la base de cette rapide propagation de la pandémie en Côte d'Ivoire. Celles-ci ont été regroupées en classes suivant les $5 \mathrm{M}$ en accord avec le diagramme d'ISHIKAWA.Conclusion : Le COVID-19 représente un réel problème de santé publique majeur en Côte d'Ivoire. Sa propagation est rapide et non maîtrisée. La population ne s'est pas encore approprié les gestes barrières. Et les causes de cette diffusion exponentielle de la pandémie ont été identifiées. Recommandations : A la lumière des résultats, certaines recommandations telles que l'automatisation des dispositifs de lavage des mains dans les lieux public, la pulvérisation régulière des lieux publics, le port obligatoire des masques, la bonne protection des aliments directement consommables ...ont été faites afin de freiner la propagation de cette pandémie en Côte d'Ivoire.
\end{abstract}

Mots-clés : COVID-19, progression, transmission, facteurs de risque, Côte d'Ivoire

\begin{abstract}
Background: Covid-19 (coronavirus disease - 19) is an infection caused by a coronavirus known as SARS-Cov2 (Severe Acute Respiratory Syndrome Coronavirus 2), a highly contagious virus that affects the respiratory tract. There are a large number of patients around the world causing many loss of human life. In Côte d'Ivoire, as everywhere else, governments and specialists are committed to the fight against covid-19. To this day, the best way to fight is prevention. Objective: To take stock of the covid-19 pandemic in Côte d'Ivoire with a view to improving the fight against it. Methods: The first step consisted of analyzing the progression of the pandemic in Côte d'Ivoire over the period from March to May 2020. The second step was the diagnosis of the mode of transmission of the pandemic. The third step consisted of researching risk factors from field surveys and analysis of the facts generally observed in Côte d'Ivoire. Results: Analysis of the progression of the pandemic shows that it is spreading randomly and at a very high rate in Côte d'Ivoire. The number of infected people is unevenly distributed across the country and over time. The results of the diagnosis of the mode of transmission of the pandemic in Ivory Coast from the control chart of averages showed that the transmission of the pandemic in the country is out of control. Research into risk factors based on field surveys and analysis of facts observed in the country reveals that several causes are at the base of this rapid spread of the pandemic in Côte d'Ivoire. These have been grouped into classes according to the $5 \mathrm{M}$ according to the ISHIKAWA diagram. Conclusions: COVID-19 represents a real major public health problem in Côte d'Ivoire. Its spread is rapid and uncontrolled. The population has not yet adopted the barrier gestures. And the causes of this exponential spread of the pandemic have been identified. Recommendations: In the light of the results, some recommendations such as the automation of hand washing devices in public places, regular spraying of public places, compulsory wearing of masks, good protection of directly consumable food, etc. have been made in order to curb the spread of this pandemic in Côte d'Ivoire.
\end{abstract}

Keywords: COVID-19, progression, transmission, risk factors, Côte d'Ivoire. 


\section{Introduction}

Le COVID-19 est une infection causée par un coronavirus (SARS-COV-2), un virus très contagieux qui affecte les voies respiratoires. Il se transmet d'une personne à l'autre personne à travers des gouttelettes respiratoires expulsées par le nez ou la bouche d'une personne infectée qui éternue ou tousse [1-2]. Il se transmet également d'un objet infecté à l'homme. Précisément lorsqu'une personne touche un objet ou une surface contaminée et qui se touche par la suite le nez, la bouche ou l'œil. Comme les personnes ne sont pas protégées contre ce nouveau virus, on compte un grand nombre de personnes infectées dans le monde [36]. La Côte d'Ivoire a enregistré son premier cas de COVID -19 le 11 mars 2020. Depuis lors, le nombre des personnes contaminées ne fait que s'accroître. Partout dans le monde, les gouvernements, les spécialistes et les populations se sont engagés dans une dynamique de lutte contre le COVID-19 [7-8]. La présente étude a fait un état des lieux de la pandémie de covid-19 en Côte d'Ivoire en vue d'en améliorer la lutte.

\section{Méthodes, résultats et discussion}

\section{1- Type et période d'étude}

Il s'est agi d'une étude exploratoire ayant porté sur la pandémie de covid-19 en Côte d'Ivoire dans la période du 11 mars au 30 juin 2020.

\subsection{Cartes de contrôle}

Les cartes de contrôle constituent un pilier de la méthode six Sigmas. Elles ont été inventées par Shewhart en 1924 [5]. C'est un document qui permet de fournir un enregistrement dynamique du processus pour agir efficacement et contrôler la constance du procédé.

\section{Objectif des cartes de contrôle}

L'objectif d'une carte de contrôle est de donner une image du déroulement du processus. Cette image doit permettre de discerner si à un moment donné, il y a la présence d'une cause spéciale ou si les variations observées ne sont dues qu'à des causes communes.

Les cartes de contrôle permettent donc de suivre la stabilité du processus au moyen de graphes et servent à :

- déterminer les moments opportuns pour un réglage éventuel ;

- connaître la capabilité du système c'est à dire le niveau de qualité qu'on peut attendre de celui-ci tant qu'il est sous contrôle ;

- stimuler l'amélioration constante de la qualité du système.

\subsection{1- Mise en place d'une carte de contrôle}

La mise en place d'une carte de contrôle se fait en trois étapes.

\section{Observation du processus}

Le but de cette étape est de connaître la variation du procédé. Il s'agit de prélever des échantillons (généralement de 5 valeurs individuelles) à des intervalles de temps réguliers. Aucun réglage n'est fait à l'intérieur d'un prélèvement (d'un échantillon). Il est nécessaire de faire au moins 20 à 25 prélèvements c'est- à-dire 20 à 25 échantillons et de calculer pour chaque échantillon, les valeurs de $\left(\bar{x}_{i}, s_{i}\right)$ ou $\left(\bar{x}_{i}, R_{i}\right)$.

\section{Calcul des limites}

Le but de cette étape est de fixer les limites générées par les causes communes. La carte de Shewhart [11] particulièrement la carte des moyennes, des écarts-types et des étendues sont utilisées pour illustrer cette partie. Le calcul des paramètres de la cartes des moyennes est donné par : A partir de $\overline{\boldsymbol{s}} L C_{\bar{x}}=v c \pm A_{3} \bar{s}$
A partir de $\overline{\boldsymbol{R}} L C_{\bar{s}}=v c \pm A_{2} \bar{R}$

Les coefficients $A_{2}$ et $A_{3}$ sont fonction de la taille de l'échantillon. On les trouve dans la table des coefficients de la carte de Shewhart. $\bar{s}$ est la moyenne des écart-types ou écart-type moyen et $\bar{R}$ l'étendue moyen.

Il faut noter que pour les procédés qui ne peuvent être centrés sur une valeur cible idéale, la cible est fixée sur la moyenne des moyennes $\overline{\bar{x}}$. Shewhart [6] a montré que lorsque les points $\bar{x}_{n}$ se trouvaient au-delà des limites de contrôle, la cause de cette dérive était un changement identifiable ou assignable (cause spéciale). D'autre part, lorsque les points $\overline{x_{2}}$ demeuraient entre les limites de contrôle, la cause de variation ne pouvait pas généralement être identifiée et le procédé est supposé maitrisé. Toutefois, il faut que la répartition des valeurs de $\bar{x}_{k}$ à l'intérieur des limites de contrôle répondent à certaines règles pour qu'il soit admis que les causes de variation des $\bar{x}_{\mathrm{n}}$ sont aléatoires c'est-à-dire que leur modèle de répartition suit une loi normale.

\subsection{2- Utilisation de la carte}

\section{Situation sous contrôle}

Un procédé est sous contrôle si :

- tous les points sont dans la limite de contrôle ;

- environ 68\% (2/3) des points sont dans le tiers central ;

- les points se répartissent au hasard de part et d'autre de la moyenne $\overline{\bar{x}}$.

\section{Situation hors contrôle}

Une situation est hors contrôle si :

- un ou plusieurs points sont hors de la limite de contrôle; il faut donc régler le procédé de la valeur qui sépare le point de la valeur cible ;

Une série de 7 points ou plus est au-dessus ou en-dessous de la valeur cible; il faut donc régler le procédé de l'écart moyen qui sépare la tendance à la valeur cible ;

- une tendance croissante ou décroissante de 7 points ou plus s'observe; il faut donc régler le procédé si le dernier point approche les limites de contrôle ;

- des points sont trop proches de la limite de contrôle ou de la valeur cible; il est donc nécessaire de confirmer cela en prélevant immédiatement un autre échantillon et l'on doit continuer la production si le point revient dans le tiers central de façon aléatoire ou régler le procédé si 
le point est également proche des limites, ou est hors contrôle ;

- tout autre modèle de variation non aléatoire se présente. Dans ce cas, l'on doit agir et régler le procédé.

\section{3- Diagramme d'ISHIKAWA}

Cette phase a consisté à rechercher les causes de la propagation de la maladie à coronavirus à partir du diagramme d'ISHIKAWA [5]. L'intérêt du diagramme d'Ishikawa [11] dans la résolution d'un problème est de regrouper les causes par famille afin d'opérer une nouvelle réflexion en abordant le problème axe par axe. La construction du diagramme d'Ishikawa est basée sur un travail de groupe; il est élaboré en plusieurs étapes qui consistent à :

- décrire clairement le problème ;

- déterminer par un brainstorming, toutes les causes possibles liées au problème de sorte que chacun donne librement ses opinions sur les origines possibles ;

- classer les causes en grandes familles : en se faisant aider des 5M (Main d'œuvre, Matière, Méthode, Milieu et Matériel), qui sont les plus utilisés, comme points de départ à la réflexion et ensuite les placer sur le squelette du diagramme d'Ishikawa [7];

- opérer une nouvelle réflexion en abordant le problème axe par axe ;

- déterminer l'impact relatif de chaque cause sur l'effet. Il s'agit de déterminer les causes probables de l'effet.

1.4. Analyse de la progression de la pandémie en Côte d'Ivoire sur la période du 11 au 30 Mars 2020

Nous avons relevé le nombre de cas journalier à l'infection de coronavirus (nombre de personnes infectées par jour) sur la période du 11 mars 2020 au 30 mars 2020. Les résultats obtenus ont permis de tracer la courbe de l'évolution du nombre des personnes déclarées infectées par jour à partir du logiciel Microsoft Excel (figure 1).

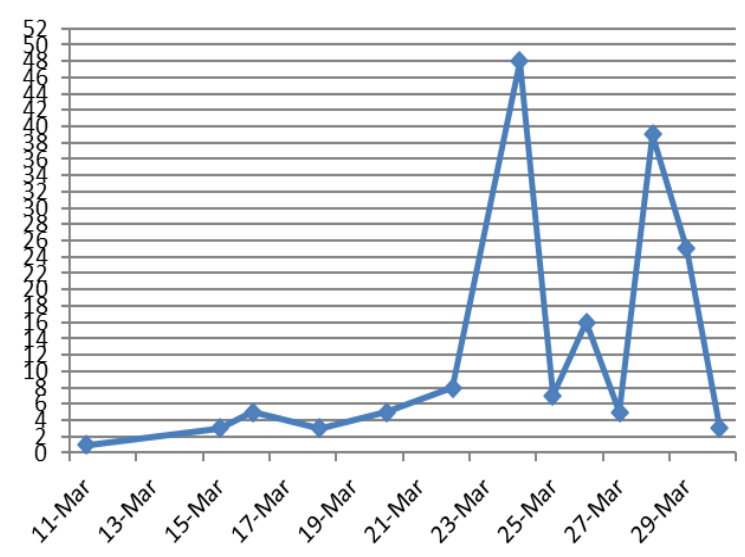

Figure 1: Visualisation du nombre de cas confirmés à covid-19 en côte d'Ivoire du 11 au 30 mars 2020.

Source : Point journalier de la pandémie sur la chaîne Radio-Télévision Ivoirienne (RTI).
Le nombre moyen $M$ de personnes infectées par jour est : $M=\frac{\sum_{i=1}^{i=N} x_{i}}{N}=12,92 . x_{i}$ désigne le nombre journalier de personnes infectées, $N$ le nombre total de personnes infectées sur la période d'étude (du 11 au 29 mars 2020).

L'analyse de cette courbe montre que la variation du nombre de personnes infectées par jour est très aléatoire. La distribution du nombre de personnes contaminées par jour ne suit aucune loi mathématique. Il est donc difficile de faire une prédiction exacte quant à la propagation de cette pandémie à coronavirus (covid-19).

Les seuls paramètres qui permettront de contrôler la propagation de la pandémie sont essentiellement: le comportement de la population, la responsabilité de chaque ivoirien, les grandes décisions des dirigeants, l'efficacité de prise en charge et de suivi des malades, la connaissance de tous les facteurs de risque pouvant entrainer la contamination d'une tierce personne. Face à ce constat, nous nous sommes engagé dans la recherche de tous les facteurs (actions, comportements, actes) même les plus élémentaires qui pourraient être à l'origine d'une quelconque contamination.

1.5. Résultats du diagnostic du système à partir de la carte des moyennes $\left(\overline{\mathbf{x}_{1}}, \mathbf{s}_{\mathrm{i}}\right)$

Il s'agit ici d'un contrôle statistique par le MSP qui est basé sur l'échantillonnage. Ainsi, vingt-cinq échantillons ont été prélevés au rythme d'un échantillon chaque 5 jours. Chaque échantillon possède 5 valeurs individuelles. En effet, une valeur individuelle n'est rien d'autre que le nombre de personnes déclarées positif au covid-19 par jour. Ces valeurs individuelles étant des grandeurs quantitatives, l'utilisation d'une carte aux mesures notamment la carte des moyennes $\left(\overline{\mathbf{x}}_{\mathbf{1}}, \mathbf{s}_{\mathbf{i}}\right)$ convient au mieux. La moyenne $\overline{\mathbf{x}_{\boldsymbol{q}}}$ et l'écart-type $\mathbf{s}_{\mathbf{i}}$ sont calculés pour chaque échantillon et sont consignés dans le tableau 1.

Tableau I : Calcul de la moyenne et de l'écart type pour chaque échantillon 


\begin{tabular}{|c|c|c|c|c|c|c|c|c|c|c|c|c|c|}
\hline \multicolumn{14}{|c|}{ ECHANTILLON } \\
\hline & E1 & E2 & E3 & E4 & E5 & E6 & E7 & E8 & E9 & E10 & E11 & E12 & E13 \\
\hline 1 & 1 & 5 & 16 & 4 & 16 & 36 & 16 & 32 & 34 & 37 & 32 & 33 & 46 \\
\hline 2 & 1 & 3 & 5 & 11 & 62 & 53 & 34 & 37 & 39 & 58 & 52 & 30 & 44 \\
\hline 3 & 2 & 8 & 39 & 4 & 26 & 41 & 54 & 36 & 14 & 29 & 55 & 127 & 48 \\
\hline 4 & 2 & 48 & 25 & 24 & 35 & 52 & 59 & 52 & 19 & 36 & 31 & 55 & 10 \\
\hline 5 & 3 & 27 & 3 & 27 & 6 & 12 & 46 & 79 & 55 & 34 & 65 & 59 & 34 \\
\hline Moyenne & 1,8 & 18,2 & 17,6 & 14 & 29 & 38,8 & 45 & 47,6 & 32,8 & 37,8 & 47,2 & 63,4 & 42,8 \\
\hline Ecarts-type & 0,57 & 19,2 & 12,6 & 14,8 & 21,4 & 21,7 & 11,9 & 18,9 & 16,5 & 11,5 & 14,7 & 37,2 & 24,6 \\
\hline \multicolumn{14}{|c|}{ ECHANTILLON } \\
\hline & E14 & E15 & E16 & E17 & E18 & E19 & E20 & & 21 & E22 & E23 & E24 & E25 \\
\hline 1 & 78 & 47 & 49 & 152 & 114 & 355 & 402 & & 70 & 285 & 310 & 302 & 366 \\
\hline 2 & 70 & 54 & 34 & 169 & 186 & 240 & 216 & & 105 & 203 & 194 & 391 & 151 \\
\hline 3 & 40 & 79 & 118 & 126 & 223 & 384 & 185 & & 205 & 290 & 228 & 323 & 142 \\
\hline 4 & 25 & 85 & 73 & 182 & 164 & 381 & 227 & & 57 & 252 & 310 & 106 & 216 \\
\hline 5 & 10 & 109 & 86 & 142 & 236 & 430 & 260 & & 13 & 218 & 246 & 165 & 207 \\
\hline Moyenne & 38,4 & 75,2 & 92,6 & 146,6 & 232,8 & 367,4 & 211,6 & & 33 & 254,6 & 256 & 270,2 & 147 \\
\hline Ecart-type & 22,7 & 24,4 & 44,8 & 28,5 & 74,1 & 73,8 & 35,4 & & 15,4 & 45,6 & 49,3 & 127,1 & 78,7 \\
\hline
\end{tabular}

Calcul des paramètres de la carte des moyennes Il s'agit de calculer la moyenne des écarts types ou écarts types moyens $(\bar{S})$; la valeur cible (fixée sur la moyenne des moyennes $(\overline{\overline{\boldsymbol{X}}})$ dans notre cas) et enfin les limites de contrôles supérieures LCS et inférieures LCI.

Valeur cible (Vc) ou moyenne des moyennes $\overline{\bar{x}}$

$$
\overline{\bar{x}}=\frac{\sum_{i=1}^{k} \bar{x}_{i}}{k}
$$

Cette valeur est calculée à partir de la formule de la relation (3) dans laquelle $\mathrm{k}$ est le nombre total d'échantillon.

Cette formule appliquée à l'ensemble des $\overline{\boldsymbol{x}}_{\boldsymbol{z}}$ contenus dans le tableau 1 permet d'obtenir : $\overline{\overline{\boldsymbol{x}}}=$ $\mathrm{Vc}=\mathbf{1 1 0 , 4 5 6}$ soit $\mathrm{Vc}=\mathbf{1 1 0 , 5}$

Ecart-type moyen ou moyenne des écarts type $(\bar{s})$

C'est la moyenne arithmétique des écarts type $\left(\mathbf{S}_{\mathbf{i}}\right)$ de chaque échantillon i. Il est obtenu par la formule de la relation (4)

$$
\overline{\boldsymbol{s}}=\frac{\sum_{i=1}^{k} \mathbf{s}_{\mathbf{i}}}{k}
$$

Ainsi, on obtient après calcul : $\overline{\mathbf{S}}=\mathbf{3 7 , 4 8 4 5 1 7 1 7}$

Limite de contrôle Supérieure LCS Cette grandeur est obtenue à partir de la formule de la relation (5).

$$
\operatorname{LCS}_{\overline{\boldsymbol{x}}}=\overline{\bar{x}}+\left(A_{3} \times \bar{s}\right)
$$

La taille de l'échantillon étant 5, la valeur de $\mathrm{A}_{3}$ correspondante est 1,427 conformément à la table des coefficients de Shewhart.

$$
\begin{gathered}
\operatorname{LCS}_{\bar{x}}=110,456+(1,427 \times 37,48451717) \\
\mathbf{L C S}_{\overline{\mathrm{x}}}=\mathbf{1 6 3 , 9 4 6 4 0 6}
\end{gathered}
$$

Limite de contrôle Inférieur LCI

Cette valeur est obtenue à partir de la formule de la relation (6)

$$
\begin{aligned}
& L C I_{\bar{x}}=\overline{\bar{x}}-\left(A_{3} \times \bar{s}\right) \\
& L C I_{\bar{x}}=110,456-(1,427 \times 37,48451717) \\
& \mathrm{LCI}_{\overline{\mathrm{x}}}=56,9655944
\end{aligned}
$$

Tracé de la carte

La carte de contrôle de la figure 2 est tracée grâce au logiciel Microsoft office Excel à partir des moyennes $\overline{\mathbf{x}}_{\mathbf{1}}$ du tableau 1 et des valeurs de LCS, LCI et Vc calculées. 


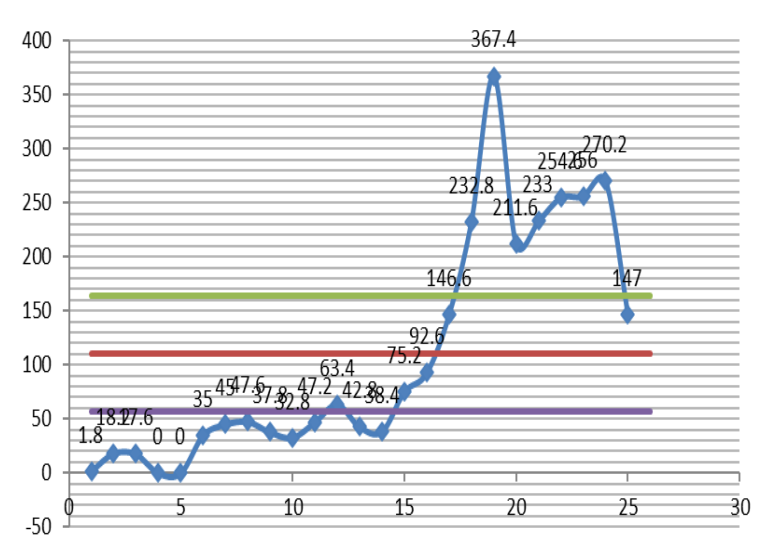

Figure 2: Carte des moyennes du nombre de personnes infectées au covid-19 par jour sur la période du 11 mars au 30 juin 2020.

\subsection{Interprétation}

Il ressort de la courbe de la figure 2 que :

- plus de la moitié des points (18 points) sont hors limites de contrôles ;

- le modèle de variation non aléatoire, une partie de la courbe ayant l'allure d'une fonction sinusoïdale. Toutes ces observations montrent que la propagation de la pandémie à covid-19 en Côte d'Ivoire est hors contrôle, non maitrisé d'où l'existence de causes spéciales ou assignables. Il est donc nécessaire d'identifier les causes potentielles (comportements et/ou attitudes favorisant cette grande propagation de la pandémie en Côte d'Ivoire) afin d'y remédier.

1.7. Identification des comportements et/ou attitudes à risque

1.7.1. Recherche des causes de contamination

Sur la période du 26 au 30 mars 2020, nous avons sillonné des banques en occurrence la Bank Of Africa (BOA), ECOBANK, Société Ivoirienne des Banques (SIB), Banque Atlantique et Société Générale de Banques en Côte d'Ivoire (SGBCI). Il $\mathrm{y}$ avait un grand nombre de personne toutefois ces banques respectaient au mieux les règles de sécurité instaurées par le gouvernement qui sont entre autres, le lavage des mains, la distance de sécurité d'au moins un mètre. Malgré le respect des règles instaurées, plusieurs clients touchent les poignets des portes des guichets pour les ouvrir et les fermer de même que les boutons de saisi des guichets automatiques pour effectuer leur opération à tour de rôle. Par ailleurs, nous avons sillonné certains marchés de Bouaké (le marché d'Ahougnanssou, le grand marché, le marché de Kôkô, le marché de Broukro) puis certains sites d'activités informelles comme les salons de coiffure, les lieux de vente d'attiéké et même la circulation routière à l'intérieur de la ville. Il ressort que :

Le lavage fréquent des mains est recommandé par les spécialistes de la santé. Ainsi devant les supermarchés, les agences et les banques, des récipients contenant de l'eau munie de vannes (une vanne par récipient) puis du savon liquide contenu dans des récipients comportant également une $\leftarrow$ - Série1 vanne par bouteille de savon sont mis à la - disposition du public. Les usagers se lavent les mains à l'entrée et quelquefois à la sortie. Un usager infecté qui actionne la vanne, peut contaminer celle-ci pendant l'ouverture et pendant la fermeture avant de s'en aller. Ainsi un usager lamda qui arrive à la suite du premier pourrait entrer en contact avec un germe, Coronavirus-19 entre autres, lorsqu'il actionne la même vanne. Concernant les téléphones portables; nous les utilisons 24h/24. Un individu ayant touché à une surface infectée par exemple s'expose d'avantage en infectant son téléphone pendant la validation d'une transaction monétaire via son téléphone. Ainsi quoiqu'il désinfecte les mains à la sortie de l'agence, il coure le risque d'une contamination lors d'une manipulation ultérieure de son téléphone. Le risque dû aux téléphones portables est assez élevé au niveau des gérants de cabine téléphonique. En effet, un téléphone d'appel ou de transfert qui est mis à la disposition des clients pour les saisies et/ou appels constitue un canal de contamination dans la mesure où, un client infecté qui touche ou communique avec ce téléphone l'infecte. Ainsi, tout client qui arrive à sa suite s'expose à une infection à coronavirus.

Un agent des forces de l'ordre et de sécurité assurant le couvre-feu ne disposant pas de dispositif de protection (gant, masque, gels hydro alcoolique,...) pourrait courir un risque de contamination en rentrant en contact direct avec une personne infectée prise par le couvre-feu.

Les produits directement consommables comme l'attiéké, les poissons grillés, les pains, les beignets et galettes vendus par une personne infectée peuvent constituer une source de contamination pour les clients. Inversement un client infecté qui touche ces aliments au cours de son achat peut transmettre le virus au commerçant ou à d'autres clients qui viendront à sa suite.

Un coiffeur ou une coiffeuse se mettant à tresser ou coiffer un client infecté pourrait s'exposer à une contamination par contact direct, par éternuement ou par la toux et inversement le client peut se retrouver contaminer par un coiffeur ou coiffeuse infecté (e).

\section{7. 2. Regroupement des facteurs de risque}

par familles selon le diagramme
d'ISHIKAWA

Des techniques d'analyse comme l'entretien et l'observation directe ont permis de recenser toutes les causes possibles de la propagation de la pandémie à coronavirus (COVID 19) et de les 
classer par famille selon les $5 \mathrm{M}$ puis de tracer le

3. diagramme d'ISHIKAWA représenté par la figure

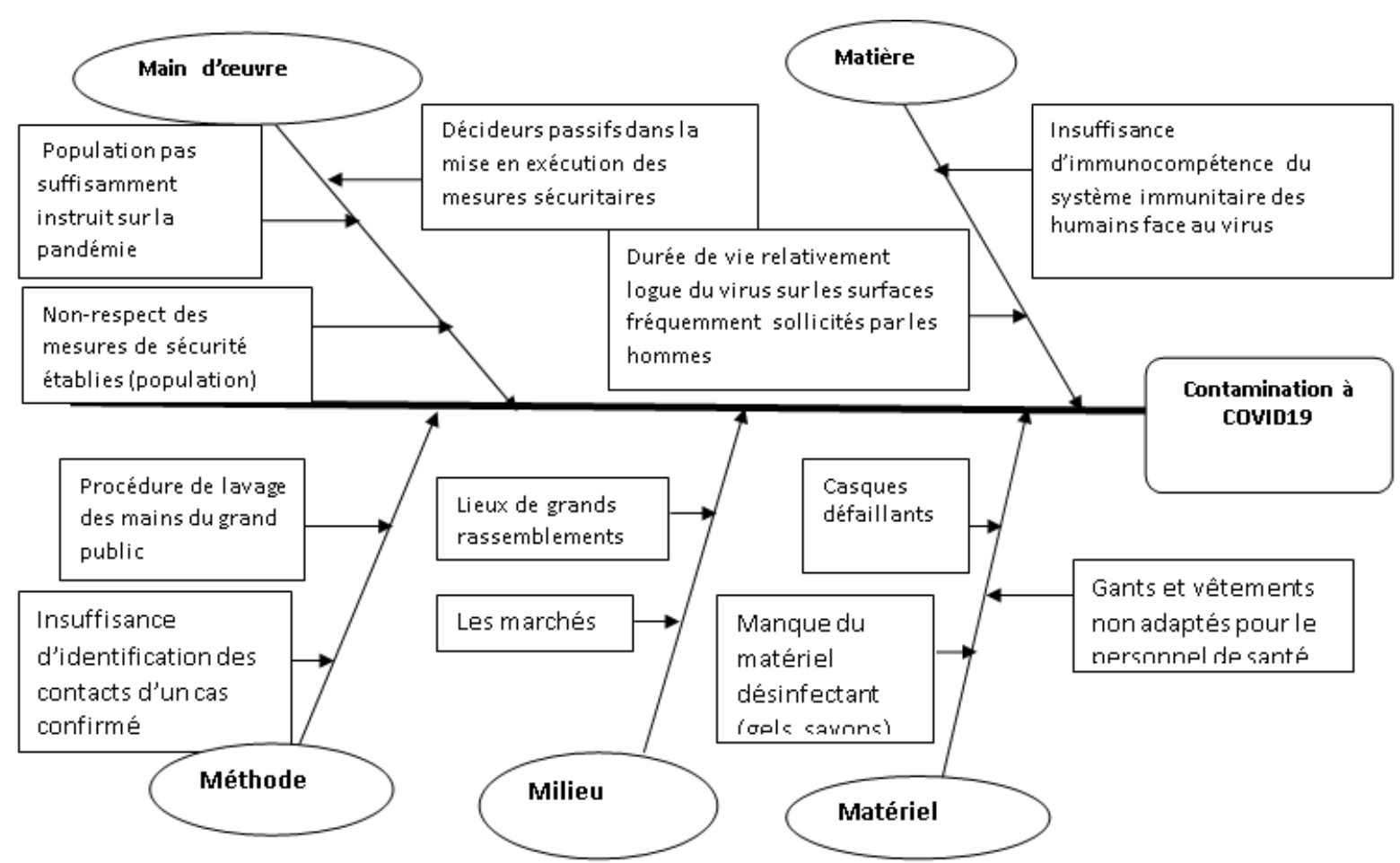

Figure 3: Diagramme d'ISHIKAWA de la propagation de la pandémie à COVID-19 en Côte d'Ivoire.

Ce regroupement des causes par famille permet de recentrer le problème et d'avoir plus d'aisances dans les prises de décision et dans la recherche de solutions.

\subsection{Discussion}

Détectée en Côte d'Ivoire le 11 mars 2020, la pandémie à coronavirus se propage sur le territoire national à la vitesse de croisière de façon aléatoire et sous un mode de transmission hors contrôle. En effet, l'analyse de la progression de la pandémie montre que celle-ci se propage de façon aléatoire et à un rythme très élevé en Côte d'Ivoire. Le nombre de personnes infectées est inégalement répartir sur l'étendue du territoire national et dans le temps. Les résultats du diagnostic du mode de transmission de la pandémie en Côte d'Ivoire à partir de la carte de contrôle des moyennes ont montré que la transmission de la pandémie dans le pays n'est pas sous contrôle. La recherche des facteurs de risque à partir d'enquêtes de terrain et d'analyse des faits observés dans le pays révèle que plusieurs causes sont à la base de cette rapide propagation de la pandémie en Côte d'Ivoire. On peut citer entre autres : le comportement de la population dans les lieux publics, la méconnaissance des voies de transmission, l'insuffisance de sensibilisation, le manque de rigueur dans l'application des mesures barrières, la gestion des produits directement consommables sur les lieux de vente, etc. Ces causes ont été regroupées en familles suivant les 5 $\mathrm{M}$ en accord avec le diagramme d'ISHIKAWA.

\section{Conclusion}

Cette étude intitulée état des lieux de la pandémie de covid-19 en Côte d'Ivoire a consisté d'abord en l'évaluation de l'ampleur et du rythme de la propagation du SARS-Cov_2 en Côte d'Ivoire puis en la recherche des facteurs de risque et enfin en des recommandations pour freiner son évolution. Il ressort que la propagation de cette pandémie en Côte d'Ivoire se fait à un rythme très élevé, de façon aléatoire et sous un mode de transmission hors contrôle. Par ailleurs, les enquêtes de terrain et l'analyse des faits observés ont montré que plusieurs causes sont à la base de cette grande propagation. Lesquelles causes ont conduit à des recommandations. En somme, nous pensons que si toutes ces recommandations sont correctement appliquées, elles contribueraient à freiner cette propagation de la pandémie à coronavirus dit $S A R S$ Cov_2 en Côte d'Ivoire.

\section{Recommandations pour freiner l'évolution de la pandémie}

A la lumière des résultats, les recommandations ciaprès peuvent être formulées :

Mettre en place une politique du type chaque citoyen son gel hydro alcoolique pour le lavage des mains puis si nécessaire, chaque citoyen son gant et son masque.

Utiliser des gants pour ouvrir les portes et manipuler les boutons des guichets, agences et 
banques puis jeter ces gants dans les poubelles aussi tôt à la sortie et désinfecter les mains afin de minimiser le risque de contamination.

Pour le lavage des mains dans les lieux publics (lieux très fréquentés), recruter une personne ou une équipe chargée à elle seule d'actionner les vannes ou pistons des gels hydro alcooliques et/ou matériels de désinfection des mains des clients. Cette dernière doit être équipée en conséquence (masques, gants, vêtements appropriés).

Automatiser les dispositifs de lavage des mains dans les lieux publics.

Assurer un nettoyage fréquent et accorder une attention particulière aux surfaces fréquemment touchées, comme les : bureaux, téléphones, ordinateurs et mains courantes.

Les domiciles, les lieux de services, les véhicules, téléphones, garde-robes des personnes déclarées positives à l'infection au SARS-Cov_2 doivent être pulvérisés par les structures compétentes en la matière.

Pulvériser régulièrement les lieux publics

Les quartiers ou zones ayant abrité un grand nombre de personnes infectées doivent être pulvérisées par les structures compétentes en la matière.

Les produits de grande consommation tels que les fruits et les légumes, les pains vendus sur le marché doivent bénéficier d'une désinfection avant d'être consommé afin de réduire le risque de propagation de la pandémie du COVID 19.

Dans les régions de grande propagation du coronavirus, l'état doit inciter la population au confinement total. Pour cela, les décideurs doivent former des équipes sur toute l'étendue du territoire national afin d'approvisionner les populations confinées en cas de besoins d'ustensiles de vie (nourriture, recharge de crédits de communication...).

Les marchés sont indispensables. Il faut cependant réglementer les marchés en disposant les commerçants de sorte à respecter au moins un mètre (distance sécuritaire) entre les commerçantscommerçants, commerçants-clients et clientsclients.

Multiplier les séances de sensibilisation sur toute l'étendue du territoire afin de faire comprendre à l'ivoirien que toutefois qu'il doit mettre la main dans le visage, la bouche ou les yeux, il doit obligatoirement désinfecter ses mains.

Faire des émissions dans le sens de rassurer la population quant à l'obtention de solution définitive à ladite pandémie et punir tout actes pouvant semer la panique et la psychose de la population.
Renforcer le dispositif de sécurité (gants, casque, gels hydro alcoolique) des forces de l'ordre lors des patrouilles pour les couvre-feux.

\section{Références bibliographiques}

1. Oumar A, Dao S, Diallo S, Kaba M, Cisse I, Tounkara A. Prévalence des infections opportunistes au cours du Sida en milieu hospitalier de Bamako, Mali. Louvain Méd 2008 ;127(1):12-7.

2. Marine G, Diana W, Juan-Manuel B, Éric M, Lionel M, Valérie P, Isabelle R, Pierre S, Yolanda M. SICOVID: un système cantonal d'information COVID pour la décision en santé publique. Rev Med Suisse 2020;16(2):2177-82.

3. Laura E, Françoise L, Thierry B. Traitements aggravant une infection par le COVID-19: vraiment ? Rev Med Suisse 2020;16(3):852-4.

4. Mamadou G, Sopi T, Affoué L, Amon B, ASSOMA, Bibata K , Nahossé Z. Évolution prévisionnelle de la pandémie du Covid-19 en République de Côte d'Ivoire : analyse statistique factuelle. Revue Afrique Science 2020;16(5):1-7.

5. Kauffmann F. Maitrise statistique des procédés. Eds. SMG; 5e édition (1 novembre 2014), Université de Caen Basse-Normandie, 2014, 82pp.

6. Kouamé L. Contribution à l'amélioration du système de stockage des produits pétroliers: cas de GESTOCI en Côte d'Ivoire. Revue Science et Technique $2020 ; 5: 354 \mathrm{~s}$

7. Jean-Yves N. Covid-19: que nous dit (ou pas) la science sur l'efficacité des masques. Rev Med Suisse 2020 ; 16 (4) : 1586-7.

8. Bertrand K. Jusqu'où plonge l'incertitude ? Rev Med Suisse 2020 ; 16 (2) : 2148-2148.

9. Christine S, Aline M, François H, Samuel P, Laurence G, Thomas F, Katharine D Véronique T, Xavier R, Clémence C, Philippe H, Marie-Claire J, Virginie P, Stephan H, Max S, Christophe G, Dina $Z$. Enjeux et défis du Covid-19 en gériatrie aiguë: leçons tirées de l'expérience genevoise. Nov ; Rev Med Suisse 2020;16(4):2153-5.

10. Jean F. Syndrome inflammatoire multisystémique associé au Covid chez l'adulte. Rev Med Suisse 2020;16(3):2146.

11. Mireille L. Statistiques de l'édition au Québec en 2013. Eds. Bibliothèque et archives nationales du Québec Dépôt légal - Bibliothèque et Archives nationales du Québec, 2015, 37 pp.

12. Mylaine $B$, Catherine $H$. La première vague de Covid-19 au Québec et les soins primaires. 2020 Nov ; Rev Med Suisse 2020;16(2):2131-4.

13. Pascal M. Que savons-nous de la transmission de SARS-CoV-2 ? Rev Med Suisse 2020 ;16(3):20789 